\title{
Hyperglycemia in the pediatric intensive care unit: Innocent bystander or villain of the piece?
}

\section{Suchitra Ranjit}

A frequent feature of critical illness is disturbed homeostasis. Thus, a central dictum in the intensive care unit (ICU) is the notion that normalizing any physiologic disturbance will hasten recovery. This belief underlines the strategy of setting physiologic goals and initiating "goal-directed therapy." [1]

Alterations in blood glucose levels are among the most frequently encountered components of disturbed homeostasis in critically ill patients, both in the adult and pediatric ICU. Stress hyperglycemia (SH) is common in critically ill and injured patients and is a component of the "fight or flight" response. Until recently, because it is so common, $\mathrm{SH}$ was considered to be a beneficial adaptive response with the raised blood glucose providing a ready source of fuel for the brain, skeletal muscle, heart and other vital organs at a time of increased metabolic demand..$^{[2]}$

On the other hand, it has been associated with complications, prolonged ICU and hospital stay and increased mortality. ${ }^{[2]}$ The important issue is whether hyperglycemia is just related to disease severity or is an independent risk factor that contributes to morbidity and mortality. If hyperglycemia is an independent risk factor, then tight glucose control (TGC) may have beneficial effects on morbidity and mortality. Conversely, if hyperglycemia is not a risk factor per se, then the risks associated with glucose control may outweigh the benefits. ${ }^{[2]}$

However, retrospective studies in adults patients have suggested that hyperglycemia was associated with an

\section{From:}

Department of Pediatric Intensive Care and Emergency Services, Apollo Children's Hospital, Chennai, Tamil Nadu, India

\section{Correspondence:}

Dr. Suchitra Ranjit, Department of Pediatric Intensive Care and Emergency Services, Apollo Children's Hospital, Chennai - 600 010, Tamil Nadu, India.

E-mail: suchitraranjit@yahoo.co.in

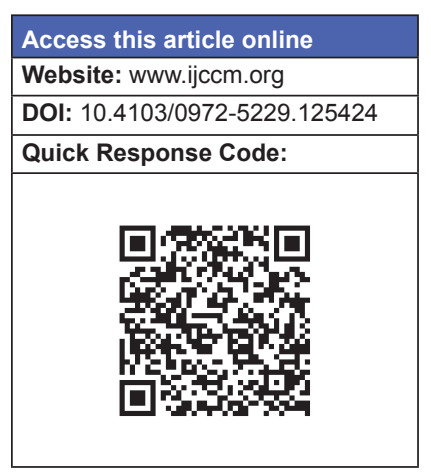

increased risk of post-operative infections and increased mortality, ${ }^{[3]}$ and in 2001, Van den Berghe et al. published the results of an innovative approach that tested a more aggressive management and proposed intensive insulin infusion therapy (IIT) targeted to tight blood glucose control $(80-110 \mathrm{mg} / \mathrm{dl}) \cdot{ }^{[4]}$

Following the van den Berghe study, some experts called for routine administration of intensive insulin therapy to normalize glucose levels in hyperglycemic patients arguing that if hyperglycemia is indeed an independent risk factor for bad ICU outcomes, then TGC may have beneficial effects on morbidity and mortality.

"IIT" or intensive insulin therapy seemed to be the magic wand that promised a favorable outcome in severely ill patients. Other studies concerning blood glucose control appeared fast world-wide and it almost felt like a crime not to target tight glycemia control in critically ill patients. ${ }^{[5]}$

However, the magic was short-lived, and benefits associated with tight blood glucose control could not be reproduced in large prospective multicenter trials. ${ }^{[2]}$ The critiques concerns regarding TGC were validated when evidence emerged from ma across the globe that the this approach carries the risk of increased frequency of hypoglycemia. Subsequently, the NICE-SUGAR study has demonstrated that moderate blood glucose control 
$(140-180 \mathrm{mg} / \mathrm{dl})$ is associated with lower mortality and a lower risk of hypoglycemia when compared with TGC. ${ }^{[6]}$

In parallel, there have been emerging concerns over the optimal glucose level in various ages and disease states, the accuracy of measurements, the impact of TGC across the heterogeneous Pediatric Intensive Care (PICU) population, the resources required to attain TGC and the risk of hypoglycemia with potentially devastating consequences. Further, it is unclear whether it is the range that is important, or limiting the variability that is required. ${ }^{[1]}$

With respect to $\mathrm{SH}$ in the pediatric ICU population, Srinivasan et al. reviewed 152 critically ill children and SH was found to be common in critically ill children. ${ }^{[7]}$ Peak blood glucose and duration of hyperglycemia were proved to be independently associated with mortality in patients.

In this issue of the Journal, Patki and Chougule in a prospective observational study, have sought to determine the incidence and association of hyperglycemia with outcome of critically ill children ${ }^{[8]}$ This study adds to the growing body of knowledge that associates the timing, intensity, duration and variability of glycemia with outcomes in critically ill children. They concluded that $69.3 \%$ of PICU children during the study had hyperglycemia (defined as blood glucose $>126 \mathrm{mg} / \mathrm{dl}$ ) and this was associated with high morbidity and mortality.

In an another recent Indian study from the pediatric ICU from all India institute of medical sciences, Bhutia et al. in their study have reported that hyperglycemia (blood glucose $>126 \mathrm{mg} / \mathrm{dl}$ ) is common in critically ill children and values $>180 \mathrm{mg} / \mathrm{dl}$ are associated with mortality. They also noted that hyperglycemia, hypoglycemia (blood glucose $<40 \mathrm{mg} / \mathrm{dl}$, and glucose variability were associated with multiple organ dysfunction..$^{[9]}$

Further muddying the waters of TGC in pediatric ICU is recent literature from the Leuven group where van den Berghe and her pediatric colleagues studied the long-term neurologic outcome of sick children in the PICU managed with TGC. ${ }^{[10]}$

TGC in the PICU (associated with more frequent episodes of hypoglycemia $(<40 \mathrm{mg} / \mathrm{dl})$ compared with usual care) did not affect full-scale IQ score and had no increased incidence of poor outcomes. Brief hypoglycemia evoked by TGC was not associated with worse neurocognitive outcome and at 4 year follow-up, children who had been treated with TGC during a PICU admission did not have a worse measure of intelligence than those who had received usual care.
A recent ongoing randomized control trial, the Heart and Lung Failure - Pediatric Insulin Titration Trial (HALF-PINT) should provide information to further guide best practice (HALF-PINT, ClinicalTrials.gov Identifier: NCT01565941).

To conclude, hyperglycemia is frequently seen in acutely ill patients and has historically been viewed as a normal response to stress. Recent evidence indicate a clear negative correlation of hyperglycemia with survival in both adult and pediatric ICUs.

In addition to recognizing and treating hyperglycemia in ICU, it is as important to identify other frequently overlooked factors that contribute to hyperglycemia, such as medications, intravenous fluids, and enteral and parenteral nutrition.

Studies demonstrate that TGC with intensive insulin therapy to achieve euglycemia may reduce mortality and morbidity in certain groups of critically ill adults and children, but equally, has the very real potential to cause hypoglycemia. The ideal goal for blood glucose in the PICU particularly in children with septic shock and neurologic compromise warrants further evaluation.

\section{References}

1. Tasker RC. Pediatric critical care, glycemic control, and hypoglycemia: What is the real target? JAMA 2012;308:1687-8.

2. Marik PE. Glycemic control in critically ill patients: What to do post NICE-SUGAR? World J Gastrointest Surg 2009;1:3-5.

3. McCowen KC, Malhotra A, Bistrian BR. Stress-induced hyperglycemia. Crit Care Clin 2001;17:107-24.

4. van den Berghe G, Wouters P, Weekers F, Verwaest C, Bruyninckx $\mathrm{F}$, Schetz M, et al. Intensive insulin therapy in critically ill patients. N Engl J Med 2001;345:1359-67.

5. Oeyen S. Do you (still) believe in tight blood glucose control? Crit Care Med 2008;36:3277-8.

6. NICE-SUGAR Study Investigators, Finfer S, Chittock DR, Su SY, Blair D, Foster D, et al. Intensive versus conventional glucose control in critically ill patients. N Engl J Med 2009;360:1283-97.

7. Srinivasan V, Spinella PC, Drott HR, Roth CL, Helfaer MA, Nadkarni V. Association of timing, duration, and intensity of hyperglycemia with intensive care unit mortality in critically ill children. Pediatr Crit Care Med 2004;5:329-36.

8. Patki VK, Chougule SB. Hyperglycemia in critically ill children. Indian J Crit Care Med 2014;18:8-13.

9. Bhutia TD, Lodha R, Kabra SK. Abnormalities in glucose homeostasis in critically ill children. Pediatr Crit Care Med 2013;14:e16-25.

10. Mesotten D, Gielen M, Sterken C, Claessens K, Hermans G, Vlasselaers D, et al. Neurocognitive development of children 4 years after critical illness and treatment with tight glucose control: A randomized controlled trial. JAMA 2012;308:1641-50

How to cite this article: Ranjit S. Hyperglycemia in the pediatric intensive care unit: Innocent bystander or villain of the piece?. Indian J Crit Care Med 2014;18:6-7.

Source of Support: Nil, Conflict of Interest: None declared. 